\title{
Supervision
}

Resümee

\section{Jung, facettenreich und mit Perspektive}

Das Themenheft zeigt, wie vielfältig das Thema Supervision zu betrachten ist: Jenseits einer historischen Sichtweise finden sich diverse theoretische und methodische Perspektiven, zahlreiche Zugangsweisen mit z.T. ähnlichen, aber auch differenten Auffassungen.

Die Entwicklungsgeschichte der Supervision stellen Möller \& Kotte in ihrem Beitrag dar: Ursprünglich eine Form der Begleitung ehrenamtlicher Sozialarbeiter, weitete sich das Verfahren Richtung Balintgruppen und psychoanalytische Kontrollsupervision, verzweigte sich in den Methoden und Schulen und umfasst heute auch die Supervision in bzw. von Organisationen.

Lässt sich heutzutage von „einem“ Verfahren bzw. „der“ Supervision sprechen? Das vorliegende Heft verdeutlicht, dass differente Supervisionsformen weiterhin und gleichzeitig bestehen - und es scheint, als habe sich keine geschichtlich überholt. Der Reigen ist immer bunter geworden, wie schon allein an ganz unterschiedlichen Supervisionsansätzen im Bereich der Psychotherapie erkennbar ist.

Auckenthaler, die Selbstverständnis und Stärken klientenzentrierter Supervision schildert, vertritt die These, dass das bewusste Herstellen von Interaktionsbedingungen zur Erleichterung reflexiver Prozesse in Fallsupervisionen maßgeblich auf den klientenzentrierten Ansatz zurückgehe. Dass die Beziehung zwischen Supervisand und Supervisor von entscheidender Bedeutung für gelingende Supervision ist, durchzieht alle Beiträge dieses Hefts. Haubl zufolge nutzen Supervisanden ihren Supervisor oder ihre Supervisorin idealtypisch als externe Reflexionsfunktion, während letztere(r) gleichzeitig die Aufgabe habe, die Reflexionsbereitschaft und -fähigkeit des Supervisanden zu stärken. Während Haubl aus psychoanalytischer Perspektive dabei die Bedeutung von „Unbewusstheit“ diskutiert, ist der Duktus im Beitrag Liebs eher methodisch geprägt. Er beschreibt Supervision als Prozess, in dem bewusst von Haupt- in Unterprogramme geschaltet werde et vice versa.

Die beiden Beiträge von Haubl und Lieb sind in diesem Heft beispielhaft dafür, wie unterschiedlich supervisorisches Denken und Handeln sich darstellt. Demgegenüber sind die Beiträge von Schigl und Ebbecke-Nohlen eher durch Verbindendes charakterisiert: Sie betonen die Notwendigkeit, in der Supervision unterschiedliche Perspektiven als gleichberechtigt zu werten und zugleich Perspektivenwechsel anzuregen, um zu Änderungen oder Lösungen zu gelangen.

In der kognitiven Verhaltenstherapie hat die Schematherapie in den letzten Jahren hohe Bedeutung erlangt. Auf die Veränderung sogenannter dysfunktionaler Muster fokussierend, kann sie als Supervisionsansatz laut Jacob \& Zens auch für andere Psychotherapierichtungen hilfreich sein. Demgegenüber befasst LinkeStillger sich in der Supervision psychotherapeutischer Prozesse mit dem Einsatz kreativer Methoden und stellt die Bedeutung von Symbolen heraus. 
Wittich und Engelhardt-Schagen lenken den Blick im Folgenden auf Supervision in Organisationen. Ihre Beiträge beleuchten anhand praktischer Beispiele aus Krankenhaus und Hochschule, dass und wie Supervision neben der Unterstützung einzelner Beschäftigter bzw. ganzer Teams auch Impulse zur Verbesserung betrieblicher Bedingungen zu geben vermag.

In den letzten Jahren hat sich Supervision aber auch neue Formate erschlossen. Dass Supervision nicht nur von Angesicht zu Angesicht möglich ist, sondern auch als „E-Beratung“ online wirksam sein kann und welche Voraussetzungen dafür nötig sind, erläutert Engelhardt in ihrem Beitrag. Aus einem besonderen und klassischen Face-to-Face-Setting wiederum, nämlich dem der Lehrsupervision, melden sich Normann und Kunde zu Wort: Als Lehrsupervisorin respektive Ausbildungskandidatin reflektieren die beiden Autorinnen Chancen und Schwierigkeiten dieser besonderen supervisorischen Arbeit und schildern jeweils ihre persönlichen Erfahrungen damit.

Diversivität in supervisorischen Prozessen ist das Thema von Steinböck. Um ausgrenzende Kategorisierungen zu überwinden, hat sich die Arbeit mit der sogenannten Diversity-Landkarte bewährt. Über Ländergrenzen hinaus blickt Knopf, wenn er sich mit Entwicklung und Perspektiven von Supervision und Supervisionsausbildungen in Europa befasst. Und um Grenzen geht es schließlich auch im Gespräch der beiden Ombudsleute Galander und Schmitz: Sie reflektieren Anlässe von und Vorgehen bei Konfliktschlichtungen zwischen Supervisanden und Supervisoren und stellen die Arbeit des Ombudsgremiums im Rahmen eines Berufsverbandes dar.

Wie geht es weiter in diesem spannenden Arbeitsfeld? Neben der Weiterentwicklung von Methoden und dem weiteren Ausbau neuer Formate muss es künftig auch darum gehen, verbindliche Richtlinien zu Kompetenzerwerb und Weiterqualifikation von Supervisoren und Supervisorinnen zu definieren. Und schließlich darum, die empirische Supervisionsforschung auszubauen und darin sowohl Fragen der Prozess- als auch der Ergebnisevaluation in den Blick zu nehmen.

Wir danken allen Autorinnen und Autoren für ihre Beiträge, die in der Zusammenschau den (sicher nicht ausgeschöpften) aktuellen Stand abbilden, aber auch offene Fragen und künftige Perspektiven in der Supervision beleuchten.

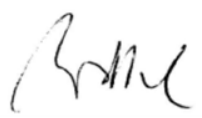

Andrea Wittich

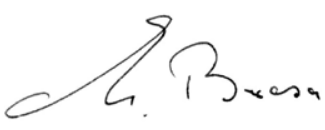

Maria Borcsa 